\title{
FROM TLS TO FE ANALYSIS: POINTS CLOUD EXPLOITATION FOR STRUCTURAL BEHAVIOUR DEFINITION. THE SAN CIRIACO'S BELL TOWER
}

\author{
R. Quattrini ${ }^{1}$, F. Clementi ${ }^{1}$, A. Lucidi ${ }^{1}$, S. Giannetti ${ }^{1}$, A. Santoni ${ }^{1}$ \\ ${ }^{1}$ DICEA, Department of Civil Engineering and Architecture, Polytechnic University of Marche - \\ (r.quattrini, francesco.clementi)@univpm.it, a.lucidi@pm.univpm.it, sarah.giannetti93@gmail.com, ariannasantoni03@gmail.com
}

\author{
Commission II, WG II/8
}

KEY WORDS: Data mining, Interoperability, Structural workflow, reality-based modelling, reliability

\begin{abstract}
:
In the last few years, the evolution of acquisition techniques allowed to acquire reality-based models increasing accurate and rich of information. On the contrary, the ability to exploit the acquired data in the most efficient, economic (in terms of invested time), and impartial way (in terms of arbitrary choices of the operator) represents the most significant gap. Interoperability theme between points cloud and informative systems becomes relevant. Excellent results were achieved in 3D data exploitation in HBIM and GIS environments, as well as in VR and AR applications, whereas the structural analysis with the Finite Element Method (FEM) still lacks robust workflows based on point clouds. The present paper proposes a methodology allowing to transform the TLS point cloud obtained from the survey directly into a 3D FEM, in a semi-automatic way and, therefore, proposes a hybrid reverse engineering approach that aims to: (i) maximizing the correspondence between the model for structural analysis and the real object; (ii) minimizing the time and the operator's decision. The strategy is validated on the belfry of the Metropolitan Cathedral-Basilica of Saint Cyriacus in Ancona, Central Italy. The reliability of the proposed model is assessed through a comparison between the model obtained from the Boolean modelling within a FEM software and the model obtained directly from points cloud processing. The comparison between the two numerical models highlights the enormous potential of the exposed method. The proposed case study shows how it is possible to develop high-quality 3D models, able to connect geometrical-historical survey with thematic analysis about structural behavior.
\end{abstract}

\section{INTRODUCTION}

Point clouds are the reference and state of art format in built heritage digitization and currently are exploited for several outputs, thanks to the integration of acquisition tools and methods: Terrestrial Laser Scanners (TLS), drones and photogrammetry. The accuracy and reliability of the data capturing for complete documentation and dissemination purposes are well recognized, also according to the economic sustainability of large survey campaign.

To take full advantage from survey data mining, the relevant focus should be on the interoperability between the starting point (points cloud) and informative systems, in particular when the same 3D model is managed to solve issues in different disciplinary fields.

On this light, recently many authors achieved excellent results in terms of 3D data exploitation. The issues of Heritage Building Information Modeling (HBIM) and the use of points cloud for the management and documentation of historical building are giving interesting research perspective and solutions. Another topic widely investigated is the world of the Information and Communication Technology (ICT) applied to the dissemination of historical buildings: Virtual Reality (VR) opens to data collected and georeferenced at various scales of definition and detail. Augmented Reality (AR) allows the visit an architecture while enjoying additional information placed next to the real physical element and Immersive Reality (IR) allows immersive journey in architecture, gathering scattered elements or remote worlds.

On the other hand, the structural analysis with the Finite Element Method (FEM) still lacks robust workflows based on point clouds. Now, the standard technique is to use the output data of the survey for the elaboration of traditional 2D drawings (plans, elevations, and sections) on which is directly based the 3D modelling in a FEM environment.

This backward path subtracts detail and precision from the model, providing approximate evaluations of the static and dynamic behavior (e.g., the non-correct inclination, the imperfection, the lack of material in the masonry walls, etc.). Moreover, this approach is time-consuming considering all the steps: definition of Level of Detail (LOD), choice of sections, modeling, meshing, application of load, analysis, etc.

The validation and assessment of new workflows related to the creation of numerical models from survey data are needed, also dealing with the challenge to perform smart and affordable modelling strategies.

The present paper proposes a methodology allowing to transform the TLS point cloud obtained from the survey directly into a 3D FEM, in a semi-automatic way.

The article is structured in five chapters. The first is about the current state of the art and analyses three macro areas involved: the acquisition of data, the previous experiences of import and use of the points cloud within a FEM and the structural analysis. The second contains a description of the case study: San Ciriaco's cathedral bell tower in Ancona. The third chapter describes the methodology applied to the case study: from the acquisition phase, through optimization of acquired data in order to be exported to the FEM environment, until the structural analysis phase. The fourth chapter provides a validation thanks to a comparison between the model obtained from the Boolean modelling within the FEM and the model obtained from the methodology applied to the case study. 


\section{STATE OF ART}

Points cloud is demonstrated as the state of art format in the recording of architectural heritage: several and robust tools are growing up in image-based techniques (Siebke et al., 2018) (Schonberger and Frahm, 2016), as well as, some years ago, was demonstrated in range based acquisitions (Bryan et al., 2013). Recently, studies focus on the exploitation of point clouds out challenging their current boundaries: in architectural heritage documentation certainly, the major challenge is to perform workflow able to give information dealing with conservation or restoration purposes. Some authors follow a possible approach of analysis on three-dimensional models in order to create a semiautomatic protocol to provide a thematic map of the decay of historical buildings (Galantucci et al., 2018). Similar approaches are possible both from workflows deriving from photogrammetric scans both from TLS data collection. Therefore, interesting elaborations were performed on 3D models, carrying out, for example, maps in false colors or morphological filters to eliminate useless information for the detection of alterations and to identify regions within a selected depth threshold value. In order to overcome the traditional and manual drawing of degradation, others describe a method of information extraction, obtained by mathematical procedures, quantitative, repeatable and verifiable (Nespeca and De Luca, 2016). Besides the algorithmic approach, it is worth to mention the graphical semiautomatic and low-cost approach (Enrico Quagliarini et al., 2017) as well as applications of Artificial Intelligence (AI) that come up. In particular, in deep learning researches and neural networks, the point clouds is nowadays an emerging data type to create a more detailed 3D geospatial information of the real world (Ye et al., 2018).

Due to the massive digitization of $\mathrm{CH}$, the data mining in a geometric survey about the heritage of rich information only a side of a single debate: the issue of $3 \mathrm{D}$ informed models. The contact point is undoubtedly the HBIM field. The HBIM approach inoculates a lot of challenges and needs, such as differences between standardization and irregularity semantic objects or unsegmented geometries as well as the presence of parametric intelligence derived from geometric accurate data. If several studies present a good exposition of many of those issues (Apollonio et al., 2016), there is a lack of structural in deep analysis in HBIM field. The main issue for HBIM studies is to solve the ontologies modeling (Acierno et al., 2017), on the contrary, the structural analysis is based on TLS data (Böttger et al., 2016) and the decay documentation is based on pictures or VR panoramas (Napolitano et al., 2018).

This work is particularly related to those cited in the following, due to similar goals in using point clouds as a source for structural analysis of masonry, with particular regard to Finite Element Analysis. The affirmation of more evolved and precise detection techniques for spatial and geometric measurements that occurred in the last decade has also aroused interest in the civil engineering community only in recent years. For the structural analysis, the given morpho-geometric data (together with the characterization of materials) represent the input data for the evaluation and analysis of the structural behaviour and acquires particular importance especially for historic masonry buildings. Specifically, the evaluation and analysis of the structural behavior that we intend to pursue are that of Finite Element Analysis (FEA) which allows discretizing the continuum in a set of finite elements interconnected by nodes. To this end, it is necessary to provide an input data that is a solid threedimensional model representative of the object to be evaluated. In recent years, more and more optimal solutions have been sought that allow us to create $3 \mathrm{D}$ models based on the points cloud. Despite there, the reality is that commonly the points cloud is not used directly to realize the computational model necessary for Finite Element Analysis.

In particular, (Caroti et al., 2012) checks the possibility of using LIDAR data to produce 3D models useful for the analysis and structural verification of buildings. This research uses the SAP2000 v.14 as structural calculation software but, to generate a model computable from such software starting from the data obtained from the points cloud, issues were found that have led to numerous elaborations and modifications of the original data. Other techniques to meshing complex geometries provide for the reconstruction of the volume by dividing it into sections and in that case the 3D model is generated by extruding along the alignment of the cross-sections (Laefer and Truong-hong, 2017), or slices, where the original 3D geometry is obtained by stacking all of the slice (Bitelli et al., 2016; Castellazzi et al., 2017, 2015). The evolution of these attempts to make the most of the data points cloud (losing the least amount of information and minimizing the number of operations performed by the operator) over the years has gradually produced more or less performing methodologies. (Tognaccini, 2009) proposes a first classification of methodologies previously pursued in the attempt to create a model based on the points cloud. (Korumaz et al., 2017) extends this classification, which states that the structural model can be obtained in five different ways.

The four methodologies proposed based on the cloud of points provide for two methodologies that indirectly use the relevant data (they provide intermediate steps that introduce a loss of information) and two others that instead take full advantage of the original data coming from the TLS (and / or photogrammetric) survey (taking into account the current computational tools and available software).

The first group includes the methodologies "CAD model based on points cloud data" and "Creating mesh Model and Curves". The first involves the generation of the model in a cad environment using primitive solids and their combinations through Boolean operations. In this approach, the points cloud is nothing more than a guide. It is superfluous to say that the presence of non-ordinary geometries or constructive defects (for example a bulging of the masonry) is sufficient so that the result is not sufficiently adherent to reality.

The second methodology while remaining modeling in a cad environment, involves the generation of the mesh from the original points cloud, which will then be used to create curves (obtained as slices of the mesh). The generated solid will, therefore, be compared to the result of the Autocad "loft" command passing through the curves generated by the mesh. Compared to the previous technique, the improvement of the obtained result is already considerable (a possible bulging of a face referred to above would be considered in this case), but the operator's decision remains high in the choice of the interval measurement between a curve and then another, and the presence of geometrically complex shapes remains difficult to represent in any case.

In the present case study, two most advanced techniques were examined, those, starting from the points cloud, generate a mesh and then convert it into NURBS (Non-uniform Rational BasisSplines) or directly into a solid model.

The approach considered the most performing from the point of view of accuracy according to (Korumaz et al., 2017) is that contemplates the creation of a mesh, from points cloud, and the subsequent conversion into NURBS.

In practice, this method requires the operator to identify significant section planes that intercept the mesh of the object and identify NURBS. These NURBS are then considered as fixed boundaries of the object by the software that will create a solid enclosed by the boundary nurbs, identified by the operator. This 
method is rather expensive from the point of view of the timing that grows depending on the complexity of the object. This is very suitable in the field of mechanical engineering where the components are constituted by the juxtaposition of simple solid elements. In the field of civil engineering it has been tested obtaining good results but with small-sized elements such as wooden beams of a roof (Bassier et al., 2016), but rather inconvenient in the case of large buildings with a higher degree of complexity.
Because of the complexity and the irregularity typical of historical masonry buildings, the approach that generates a mesh from the points cloud and then converts it to a solid object (through software that supports this operation) is the most useful (the methodology defined "Create mesh and solid model" from (Korumaz et al., 2017)).

According to the description above, the schematization proposed by (Korumaz et al., 2017) is modified taking into account the further discriminant of the operator's judgment (Figure 1).

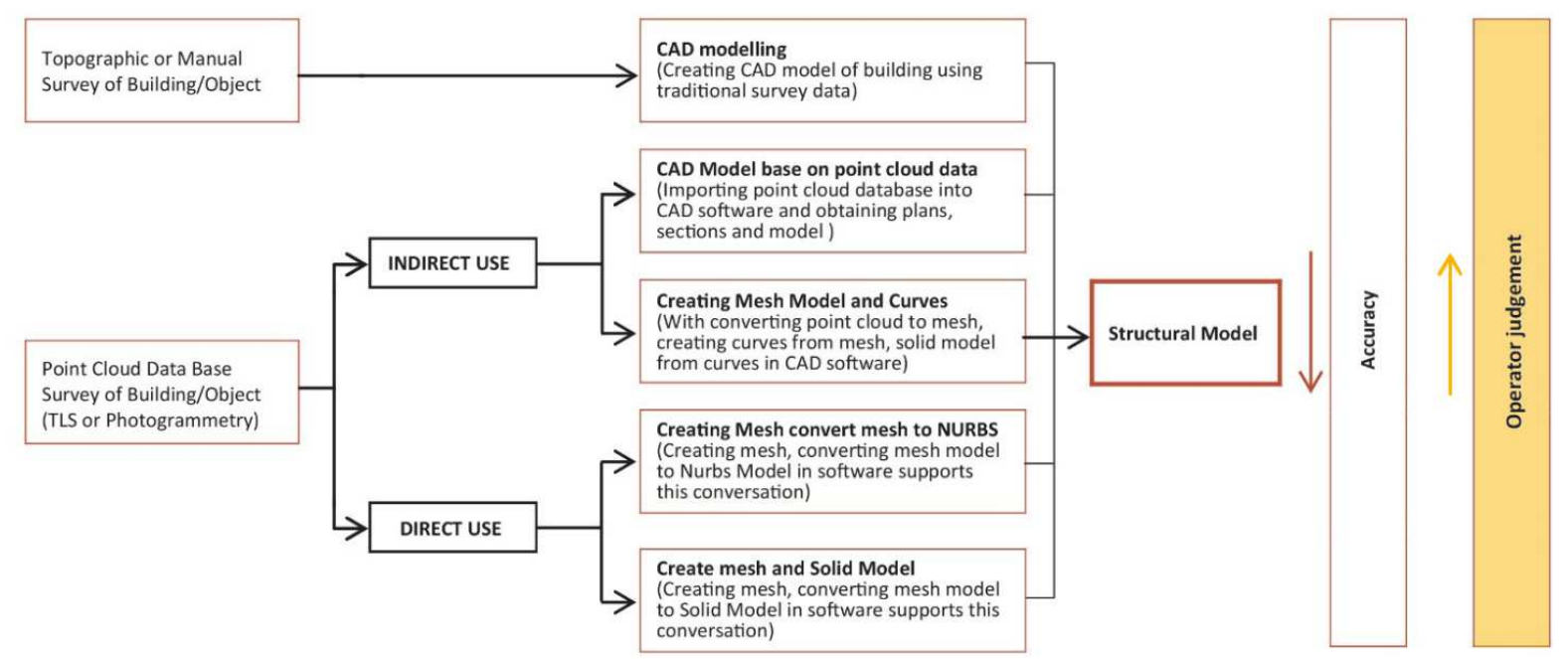

Figure 1 - Classification of methods for the generation of structural models

In line with the "Create mesh and solid model" methodology, the study of (Visintini and Spangher, 2014) exploited the points cloud of a small-sized object (a Roman statue) to generate a model used directly within Rhinoceros software through the Scan $\&$ Solve tool. This function made it possible to consider the entire volume with many $\mathrm{XY}, \mathrm{XZ}$ and $\mathrm{YZ}$ planes that will generate "cubes" on which the differential equilibrium equations will vary, different from those belonging to the domain (internal to the object) or surrounding it (on the surface of the object).

The level of detail (LOD) of the discretization of the mesh is an element of great importance for obtaining a structural analysis that is more faithful to the real behaviour of the structure (Spangher and Visintini, 2015).

For modern structures, with new industrial materials (reinforced concrete, steel, etc.), the development of a reliable mathematical model is possible, due to the fact that materials and structural elements are more uniform and sufficiently well-known (Pierdicca et al., 2016; E. Quagliarini et al., 2017). Conversely, the seismic behaviour of old masonry structures is particularly difficult to be examined. It depends on many factors such as material properties, geometry of the structure, stiffness of the floors (diaphragm effect) and connection between orthogonal walls and structural and non-structural elements (Betti and Vignoli, 2008).

One key point is the material behaviour modelling. Masonry is a composite material obtained joining natural or artificial bricks by means of mortar layers. The non-uniformity of the mixture is due to the variability of both components, mortar, and bricks, that can vary very much from place to place due to different local construction technologies. For this reason, the stiffness and the resistance have a great dispersion, but it could be useful to characterize the masonry material of ancient constructions by means of average values provided in codes or manuals (Angelillo, 2014).
Furthermore, in addition to the heterogeneous nature of masonry, also the nonlinear response, often already triggered at low deformation levels, is a challenge for structural engineering, both from a scientific and from a professional point of view. Indeed, the formulation of models for reproducing the complex nonlinear mechanical behaviour of the masonry is an active research field. Experience shows that masonry mechanical behaviour is dominated by the nonlinear phase, characterised by cracks opening, dissipative and brittle behaviour with a softening branch. Nonlinear anisotropic constitutive laws are required and behaviour in tension and in compression is markedly different. Much focus is currently given to the formulation of nonlinear constitutive relationships for masonry and a large number of models are now available (Berto et al., 2002).

The most natural approach for describing the mechanical behaviour of masonry structural elements is the adoption of continuous material models. In this framework, a wide variety of continuous 2D and 3D nonlinear models have been proposed. These include complex nonlinear mechanisms such as frictionplasticity, cohesion, crushing, damage and so on (Clementi et al., 2016).

From a numerical point of view, elastic-plastic analyses may be used to simulate masonry nonlinear behaviour. However, they fail to simulate the crack formation and brittle behaviour when the material enters the softening regime. Limit analysis methods have been frequently applied in order to investigate the collapse mechanism of masonry structures subjected to given load distributions. Smeared crack approaches and/or damage models may be used to simulate the local loss of strength masonry material suffers when it enters the nonlinear behaviour (Clementi et al., 2017). However, to the best of the authors' knowledge, the numerical analysis itself is still very demanding, especially when dealing with large and complex structures. For the sake of completeness, we mention that an alternative to modelling masonry as a homogenised continuum is the discrete element approach, which models the structure as an assembly of blocks 
with suitable interface laws (Clementi et al., 2019; Ferrante et al., 2019).

\section{CASE STUDY}

The strategy is validated with an application to the belfry of the Metropolitan Cathedral-Basilica of Saint Cyriacus in Ancona, Central Italy (Figure 2).

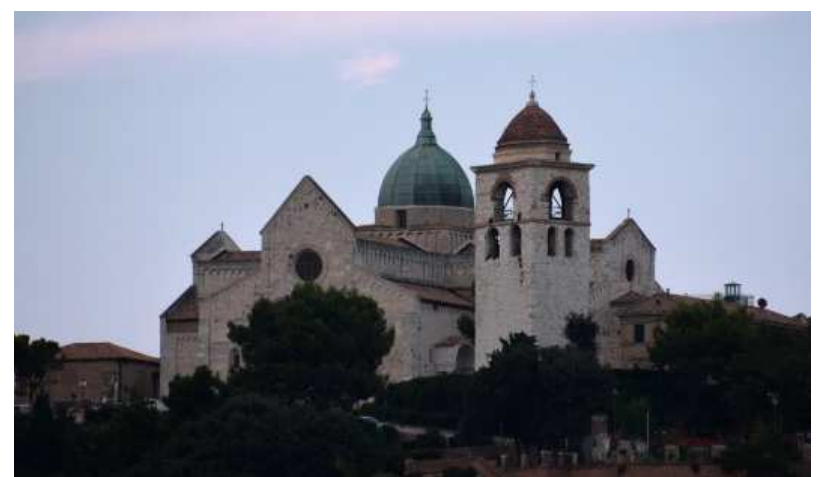

Figure 2 - Hill Metropolitan Cathedral-Basilica of Saint Cyriacus view from the Port of Ancona

The tower stands on top of the Guasco's hill and is isolated from the Church. The bell tower was probably built starting from a former military tower belonging to the defensive walls system in the top of the hill. The tower has a rectangular transversal section that remains unchanged up to the top. The roof starts from a cornice where an elliptical dome with an octagonal base is disposed.

\section{METHODOLOGY}

\subsection{Data collection}

The data acquisition campaign was carried out with a TLS Leica Scan Station P40, including the entire area of the complex. 29 standpoints were acquired, among which it is possible to count: (i) a frontal one at the entrance to the tower that allows alignment of the internal and external acquisitions, (ii) ten in the interior of the belfry (Figure 3), (iii) and eighteen external (Figure 4).

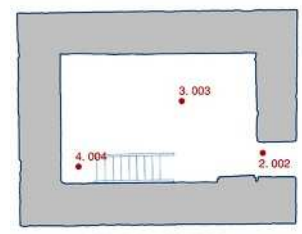

PIANTAPIANO TERRA

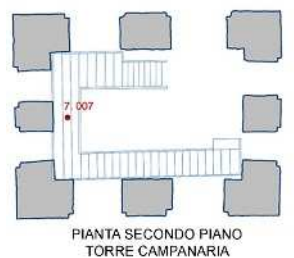

Figure 3 - Internal stations in San Ciriaco's belfry

The whole cloud, including the external square, resulting from the alignment of the 29 stations, has a maximum error of $4 \mathrm{~mm}$ ( $3 \mathrm{~mm}$ due to the instrument and $1 \mathrm{~mm}$ due to the alignment).

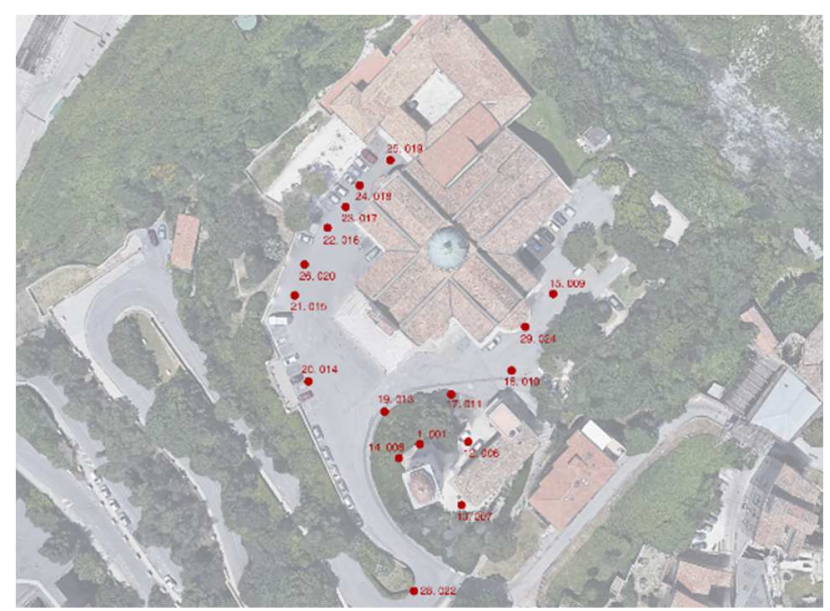

Figure 4 - External stations in the complex of San Ciriaco

Then the complete cloud was cut to isolate the tower to use it as the basis of the structural model (Figure 5).

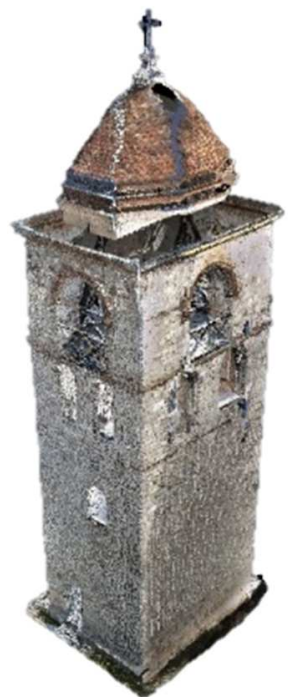

Figure 5 - Points Cloud of the tower

\subsection{Workflow (optimization)}

4.2.1 The result of the data acquisition, alignment and registration phase represents the input data of a process that will lead to the generation of a model useful for structural analysis in FEM ambient. This process involves the use of multiple programs to achieve the final 3D Solid FEM; the optimization workflow is summarized in Figure 6.

4.2.2 Operations in Geomagic $\odot$ : The most expensive operations are carried out within the Geomagic $(5)$ software. The first operation is model cleaning from every non-structural elements (steel stairs, belfry, railings, etc). At the end of this operation is obtained a cloud of 1995824 points. Next step is represented by the transformation of the points cloud into a surface mesh.

Mesh obtained is an open surface because there are missing masonry portions that during the acquisition were covered by non-structural elements that were removed in the previous phase. Missing mesh parts are restored through the command "fill hole" present in the software.

For interoperability issues, it is then necessary to execute the "Mesh doctor" command from Geomagic $(\subset$ that automatically solves geometry issues (such as auto-intersections, presence of peaks, small holes ...) that would make the file unreadable in different software, if not repaired. 

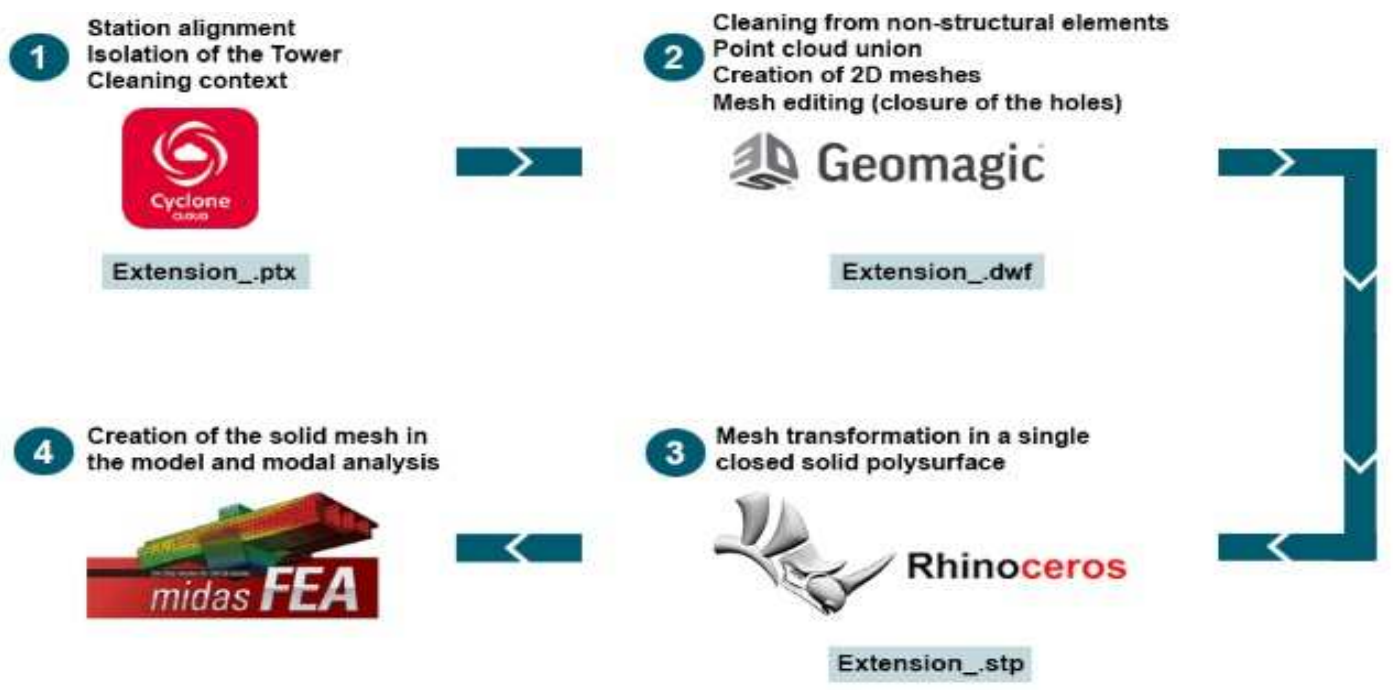

Figure 6 - Workflow from the point cloud to Midas FEA@ software

This command, however, tends to simplify and excessively uniform the geometry for the purposes useful to our study (rightangles corners are excessively rounded) then, preliminary to the command of "mesh doctor", the edges are fixed (at the corners). (Figure 7.a)

Currently, the number of meshes of the model is a limit for almost all computational software, therefore it is necessary to reduce the number of triangles, that constitutes mesh, through the decimation operation.

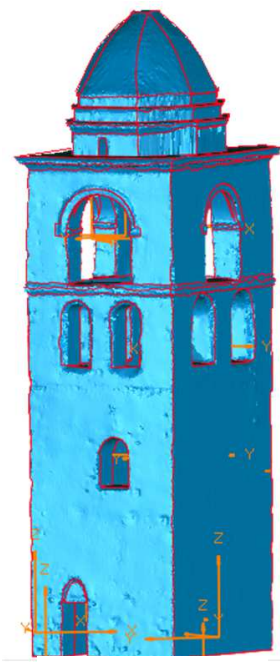

a)

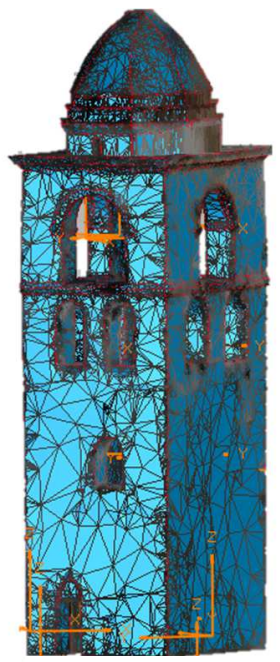

b)

We proceed by attempts until we find the model with the major number of meshes that has good interoperability with the other two software, Rhinoceros and Midas FEA@: starting from 30,000 triangles test, tests proceeded to $50,000,60,000,100,000$ up to 120,000 triangles. After the model correction, the attempts to import into software dedicated to structural analysis are excellent for decimations up to 100,000 meshes (Figure 7.b), while they do not provide results for the model with the decimation of 120,000 triangles.

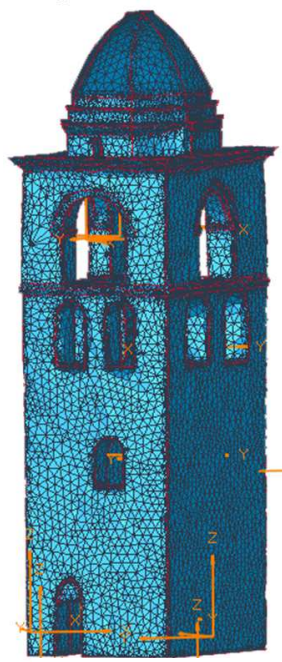

c)

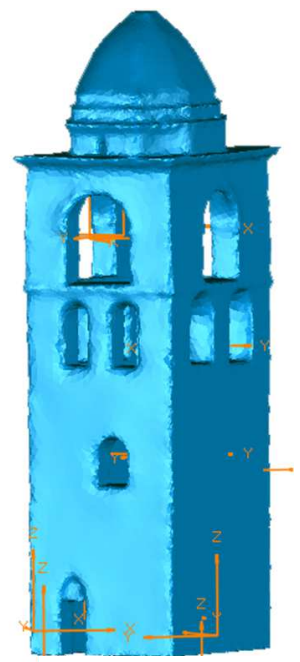

d)

Figure 7 - Mesh evolution inside Geomagic $($ software:

a) Mesh with red edges that will not be modified during the editing operations of the mesh; b) Decimated mesh of 100000 triangles; c) Mesh with regular shapes and dimension triangles; d) Final mesh (62 684 triangles)

Once the model of the bell tower has been decimated, the Mesh Doctor correction is carried out again.

Thanks to the first attempts made in Midas@ it was observed that, with solids modeled in this software environment, the object is subdivided into 3D regular elements. In order to make the model more practical and to semplify the computational work of creating a $3 \mathrm{D}$ mesh in Midas FEA $\odot$, it is necessary to regulate the shape and size of the $2 \mathrm{D}$ meshes into the Geomagic $(\mathrm{C}$ software.

The command "Recreate Mesh" converts coarse polygonal meshes in uniform meshes, it creates triangles of very regular shape. (Figure 7.c)
As already mentioned above, the Midas FEA $@$ software does not well support sharp edges of mesh, so all the red boundaries, created to avoid excessive approximation during the phases of correction, are deleted. Afterward, the regularization and the smoothing of the model are mandatory for the export in FEA (QuickSmooth).

At the end of all the operations listed above, a model consisting of 62.684 mesh is obtained. (Figure 7.d)

Once the operations in Geomagic (C) (Figure 8) have been completed, the model is exported in .stl format (binary), compatible with the Rhinoceros software. 

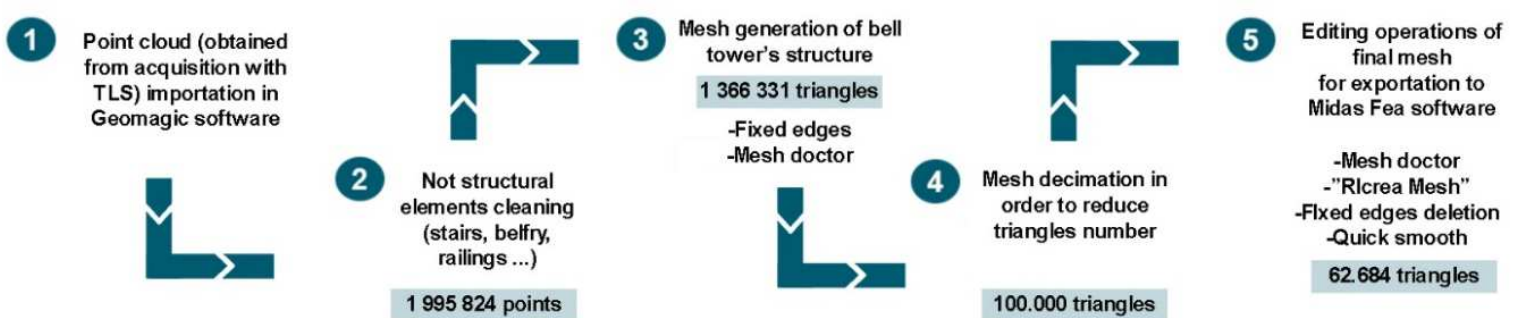

Figure 8 - Operations in Geomagic $@$ software

4.2.3 Operations in Rhinoceros: the purpose is to obtain a solid model starting from the closed surface mesh obtained from the operations performed in Geomagic $($ ).

This step is possible thanks to the Rhinoceros software that plays the role of a bridge between Geomagic $\odot$ and Midas FEA®.

So .stl file is imported into Rhinoceros which identify the object imported as a mesh. With the command "MeshANurb" the mesh is transformed into a closed polysurface ready to be exported.

The closed polysurface is exported with extension STEP (.stp) which is one of the formats identified by the Midas@ $@$ software for the import of models.

4.2.4 Import into Midas FEA $\odot$ : model is imported into the Midas FEA@ software in the modeler environment.

The imported .stp file is identified as a solid, 3D mesh generator integrated into the software creates 3D mesh made up of solid tetrahedrons that fill object volume.

"Auto-Mesh" command, in less than three hours, generated a three-dimensional mesh consisting of 251.799 elements and 60.256 nodes.

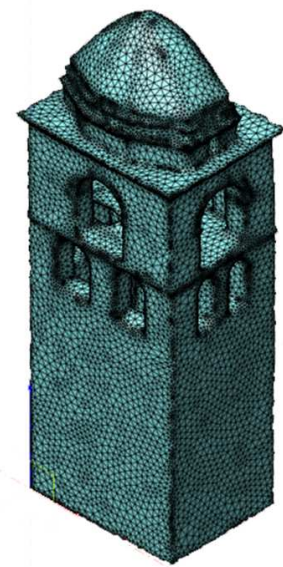

Figure 9 - Mesh generated into Midas FEA@ software

\subsection{Modal analysis}

Obtained the model on which to perform the simulations and structural analyses required, the constraints and loads were assigned to the model: the weight of the bell tower and weight of the elements that constitute it.

For a first analysis of the results with the imported model, it was decided to use not-divided model applying to it only one material: "calcarenite", the prevailing material in the construction. Once the loads have been applied, evaluation of seismic combination was carried out in order to identify: the most significant modes of vibration, the period of oscillation and the relative modal mass participant.

4.3.1 Modal analysis of the one-material model: first, a single material characterized the model: "calcarenite".

In particular, the most significant of main ways of vibrating for each direction have been identified:

- DIRECTION X: mode 2 with participant mass $54.50 \%$

- DIRECTION Y: mode 1 with participant mass $53.78 \%$

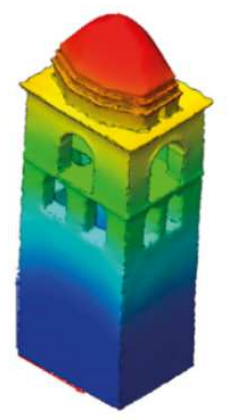

DIRECTION Y: mode 1 Participant mass $53.78 \%$

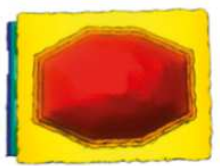

Figure 10 - Main mode shapes of vibration of one material model

4.3.2 Modal analysis of the two-material model: subsequently, the operations were repeated for the model that considers the floor of the belfry in reinforced concrete and other parts in "calcarenite".

The most significant of main ways of vibrating for each direction have been identified:

- DIRECTION X: mode 2 with participant mass $54.75 \%$

- DIRECTION Y: mode 1 with participant mass $53.95 \%$

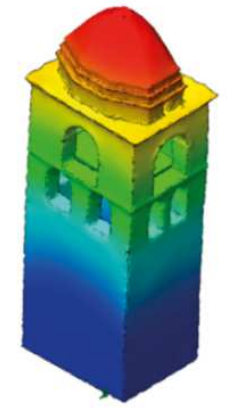

DIRECTION Y: mode 1 Participant mass $53.93 \%$

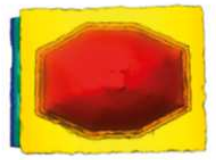
Figure 11 - Main mode shapes of vibration of two materials model
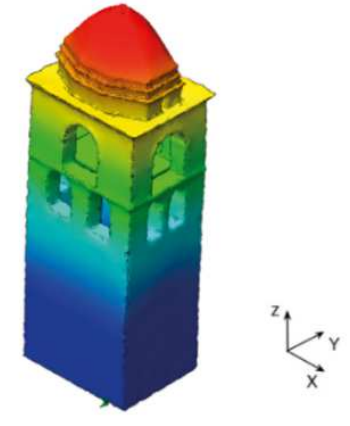

DIRECTION X: mode 2 Participant mass $54.75 \%$
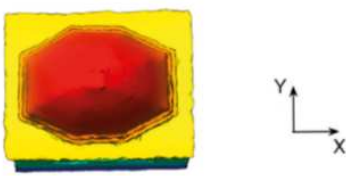


\section{RESULTS AND DISCUSSION}

The main innovation of the present work is the processing of TLS data to generate a model useful for structural analysis. Several studies try to transform three dimensional points clouds in FE models but, in most cases, the output is simple or dramatically simplified. This work constitutes a first step in validating and disseminating workflows for structural models not manipulated by the operator's judgement. In the proposed approach, the model successfully maintains metric information, with a well-adjusted Level of Detail. Moreover, compared with similar workflow (Figure 6), it is worth to mention that a complex transformation of the whole mode (inside and outside) was obtained.

On the other hand, even though the proposed method is successful, issues remain related to the complexity of the TLS model. In general, a good balance between model simplification and interoperability problems was obtained. Moreover, the benefits of the model generated by laser acquisition certainly bring improvements in time and costs, as it will no longer be necessary to rebuild the structure, increasing errors occurrence. To validate the model obtained from points cloud, two models were compared: the one deriving from points cloud and FEM model realized in Midas FEA.

A discussion on the results is provided below. The first four modes of vibrating the structure have been defined, paying attention to the modes in the 2 main directions in plan and extracting the modal plane masses. This was done for the onematerial and two-material model.

This analysis highlights the FEM model has greater usability within the program, because less complex, but it must be emphasized that it arises from a severe simplification of the model.

On the opposite, the TLS model, maintaining a high level of detail, is a very complex model, so it does not allow to perform some operations necessary for the creation of a more defined model from the material point of view.

Comparing the two models, the same mode shapes of vibration are founded. This is a comfortable result because it means that the two models have the same dynamic behaviour. Quantitatively it will be necessary to calibrate the two models comparing them with the OMA measurements, in order to evaluate which of the two models is able to get closer to the experimental measurements. The Operational Modal Analysis (OMA) is a monitoring technology able to estimate the same modal parameters (shape, frequency...) of the traditional known techniques. The basic idea is to do a modal analysis without knowing and/or controlling the excitation of inputs.

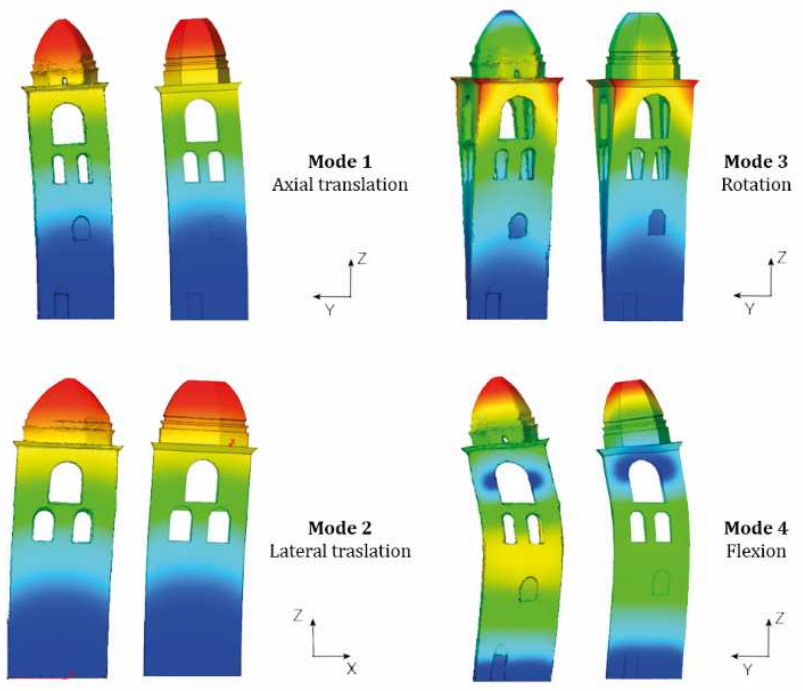

Figure 12 - Modal forms of the two models
The following tables show the frequency values for both models and the differences found between them.

\begin{tabular}{|c|c|c|}
\hline $\begin{array}{c}\text { Frequency Midas } \\
\text { Boolean model }\end{array}$ & $\begin{array}{c}\text { Frequency TLS } \\
\text { model }\end{array}$ & $\boldsymbol{\Delta f}$ \\
\hline 2,41924 & 2,56871 & $-6,18 \%$ \\
\hline 3,00398 & 3,16909 & $-5,50 \%$ \\
\hline 5,96723 & 6,21654 & $-4,18 \%$ \\
\hline 8,48928 & 8,61142 & $-1,44 \%$ \\
\hline
\end{tabular}

Table 1 - Frequency for one material models

\begin{tabular}{|c|c|c|}
\hline $\begin{array}{c}\text { Frequency Midas } \\
\text { Boolean model }\end{array}$ & $\begin{array}{c}\text { Frequency TLS } \\
\text { model }\end{array}$ & $\boldsymbol{\Delta f}$ \\
\hline 2,41924 & 2,56871 & $-6,18 \%$ \\
\hline 3,00398 & 3,16909 & $-5,50 \%$ \\
\hline 5,96723 & 6,21654 & $-4,18 \%$ \\
\hline 8,48928 & 8,61142 & $-1,44 \%$ \\
\hline
\end{tabular}

Table 2 - Frequency for two materials models

With the present work it has been verified how, with the TLS model, despite some questions still to be resolved, it is possible to obtain good results to understand the structural behavior of this type of towers.

\section{CONCLUSION}

Within the present work, it is shown a way of data mining on TLS survey: a primary goal was indeed to add value to the points cloud out of the boundaries of morphometric and chromatic type. Similar works are able to enrich the digital documentation tools and output with great potential, in the light to allow structural assessments. This last perspective is fundamental for EU territories and has a strong social impact for the protection of the historical heritage, especially for structures that are most affected by the seismic action. For this reason, the validation of the strategy has been performed on a bell tower, which embodies all the typical complexities of monumental historical buildings.

In order to increase the level of detail and precision, a successful aim in this research has been to obtain a model that is high quality reality-based, enabling also further development to 4D. In particular, as future work, similar models should be enriched by data describing in real time deformations and/or displacements. After a series of optimized steps, a model was imported to the Midas FEA $\odot$ software in order to perform the simulations and structural analysis: a sufficient level of interoperability was obtained. This was done previously by inserting a single material, in order to have a first general view of the modal forms and the most significant ways of vibrating.

The obtained model, however, due to the high complexity, cannot be divided into several elements to which assign different materials. For this reason, subsequently, a second model was generated inside Midas FEA@ software. Inside its slab and beams in reinforced concrete were characterized with appropriate material, so in this way, a further result was obtained.

Transforming laser scanning data into a 3D model that can be used for structural analysis, is emerging as a viable pathway to obtain models that can be used in structural calculation software. Future works may try to overcome the interoperability problems encountered, so to have lower calculation times and to have models more responsive to reality with metric information of great detail.

\section{REFERENCES}

Acierno, M., Cursi, S., Simeone, D., Fiorani, D., 2017. Architectural heritage knowledge modelling: An ontology-based framework for conservation process. J. Cult. Herit..

https://doi.org/10.1016/j.culher.2016.09.010 
Angelillo, M., 2014. Mechanics of Masonry Structures, CISM International Centre for Mechanical Sciences. Springer Vienna, Vienna. https://doi.org/10.1007/978-3-7091-1774-3

Apollonio, F.I., Gaiani, M., Sun, Z., 2016. A Reality Integrated BIM for Architectural Heritage Conservation. Handb. Res. Emerg. Technol. Archit. Archaeol. Herit., p. 31.

Bassier, M., Hadjidemetriou, G., Vergauwen, M., Van Roy, N., Verstrynge, E., 2016. Implementation of Scan-to-BIM and FEM for the Documentation and Analysis of Heritage Timber Roof Structures. In: Lecture Notes in Computer Science (Including Subseries Lecture Notes in Artificial Intelligence and Lecture Notes in Bioinformatics), pp. 79-90.

https://doi.org/10.1007/978-3-319-48496-9_7

Berto, L., Saetta, A., Scotta, R., Vitaliani, R., 2002. An orthotropic damage model for masonry structures. Int. J. Numer. Methods Eng., Vol. 55, pp. 127-157. https://doi.org/10.1002/nme.495

Betti, M., Vignoli, A., 2008. Assessment of seismic resistance of a basilica-type church under earthquake loading: Modelling and analysis. Adv. Eng. Softw., Vol. 39, pp. 258-283.

https://doi.org/10.1016/j.advengsoft.2007.01.004

Bitelli, G., Castellazzi, G., D'altri, A.M., De Miranda, S., Lambertini, A., Selvaggi, I., 2016. Automated voxel model from point clouds for structural analysis of cultural heritage. In: International Archives of the Photogrammetry, Remote Sensing and Spatial Information Sciences - ISPRS Archives.

https://doi.org/10.5194/isprsarchives-XLI-B5-191-2016

Böttger, H.M., Arce Bazán, C.J., Saarman, N.P., 2016. 3D laser scanning for preservation and structural monitoring of historic California adobe missions. J. Green Build., Vol. 11, pp. 1-14. https://doi.org/http://dx.doi.org/10.3992/jgb.11.4.1.1

Bryan, P., Blake, B., Bedford, J., Mills, J., 2013. Metric Survey Specifications for Cultural Heritage.

Caroti, G., Franconi, A., Piemonte, A., 2012. Metodologia di elaborazione di dati laser scanner per la generazione di modelli utili al calcolo strutturale. In: Atti $16 a$ Conf. Naz. ASITA 2012, pp. 383390.

Castellazzi, G., Altri, A.M.D., Miranda, S. De, Ubertini, F., 2017. An innovative numerical modeling strategy for the structural analysis of historical monumental buildings. Eng. Struct., Vol. 132, pp. 229248. https://doi.org/10.1016/j.engstruct.2016.11.032

Castellazzi, G., D’Altri, A., Bitelli, G., Selvaggi, I., Lambertini, A., 2015. From Laser Scanning to Finite Element Analysis of Complex Buildings by Using a Semi-Automatic Procedure. Sensors, Vol. 15, pp. 18360-18380. https://doi.org/10.3390/s150818360

Clementi, F., Ferrante, A., Giordano, E., Dubois, F., Lenci, S., 2019. Damage assessment of ancient masonry churches stroked by the Central Italy earthquakes of 2016 by the non-smooth contact dynamics method. Bull. Earthq. Eng.

https://doi.org/10.1007/s10518-019-00613-4

Clementi, F., Gazzani, V., Poiani, M., Lenci, S., 2016. Assessment of seismic behaviour of heritage masonry buildings using numerical modelling. J. Build. Eng., Vol. 8, pp. 29-47.

https://doi.org/10.1016/j.jobe.2016.09.005

Clementi, F., Quagliarini, E., Monni, F., Giordano, E., Lenci, S., 2017. Cultural Heritage and Earthquake: The Case Study of "Santa Maria Della Carità" in Ascoli Piceno. Open Civ. Eng. J., Vol. 11, pp. 1079-1105. https://doi.org/10.2174/1874149501711011079

Ferrante, A., Clementi, F., Milani, G., 2019. Dynamic Behavior of an Inclined Existing Masonry Tower in Italy. Front. Built Environ., Vol. 5. https://doi.org/10.3389/fbuil.2019.00033
Galantucci, R.A., Fatiguso, F., Galantucci, L.M., 2018. A proposal for a new standard quantification of damages of cultural heritages, based on 3D scanning. SCIRES-IT, Vol. 8, pp. 121-138. https://doi.org/10.2423/i22394303v8n1p121

Korumaz, M., Betti, M., Conti, A., Tucci, G., Bartoli, G., Bonora, V., Fiorini, L., 2017. An integrated Terrestrial Laser Scanner ( TLS ), Deviation Analysis ( DA ) and Finite Element ( FE ) approach for health assessment of historical structures . A minaret case study. Engineering Structures, Vol. 153, pp. 224-238. https://doi.org/10.1016/j.engstruct.2017.10.026

Laefer, D.F., Truong-hong, L., 2017. Automation in Construction Toward automatic generation of 3D steel structures for building information modelling. Autom. Constr., Vol. 74, pp. 66-77. https://doi.org/10.1016/j.autcon.2016.11.011

Napolitano, R.K., Scherer, G., Glisic, B., 2018. Virtual tours and informational modeling for conservation of cultural heritage sites. $J$. Cult. Herit., Vol. 29, pp. 123-129. https://doi.org/10.1016/j.culher.2017.08.007

Nespeca, R., De Luca, L., 2016. Analysis, thematic maps and data mining from point cloud to ontology for software development. ISPRS - Int. Arch. Photogramm. Remote Sens. Spat. Inf. Sci., Vol. XLI-B5, pp. 347-354. https://doi.org/10.5194/isprsarchives-XLIB5-347-2016

Pierdicca, A., Clementi, F., Maracci, D., Isidori, D., Lenci, S., 2016. Damage detection in a precast structure subjected to an earthquake: A numerical approach. Eng. Struct., Vol. 127, pp. 447-458. https://doi.org/10.1016/j.engstruct.2016.08.058

Quagliarini, Enrico, Clini, P., Ripanti, M., 2017. Fast, low cost and safe methodology for the assessment of the state of conservation of historical buildings from 3D laser scanning: The case study of Santa Maria in Portonovo (Italy). J. Cult. Herit., Vol. 24, pp. 175-183. https://doi.org/10.1016/j.culher.2016.10.006

Quagliarini, E., Maracchini, G., Clementi, F., 2017. Uses and limits of the Equivalent Frame Model on existing unreinforced masonry buildings for assessing their seismic risk: A review. J. Build. Eng., Vol. 10, pp. 166-182. https://doi.org/10.1016/j.jobe.2017.03.004

Schonberger, J.L., Frahm, J.-M., 2016. Structure-from-Motion Revisited. In: 2016 IEEE Conference on Computer Vision and Pattern Recognition (CVPR). IEEE, pp. 4104-4113. https://doi.org/10.1109/CVPR.2016.445

Siebke, I., Campana, L., Ramstein, M., Furtwängler, A., Hafner, A., Lösch, S., 2018. The application of different 3D-scan-systems and photogrammetry at an excavation - A Neolithic dolmen from Switzerland. Digit. Appl. Archaeol. Cult. Herit., Vol. 10. https://doi.org/10.1016/j.daach.2018.e00078

Spangher, A., Visintini, D., 2015. Il livello di dettaglio dei modelli 3D geomatici per l'analisi strutturale FEM. Atti 19a Conf. Naz. ASITA 2015, pp. 755-762.

Tognaccini, R., 2009. La Chiesa di Santa Maria del Mar a Barcellona - Dal Rilievo Tridimensionale all'Analisi Strutturale. University of Pisa.

Visintini, D., Spangher, A., 2014. Il contributo della Geomatica per l'analisi strutturale dei beni culturali : l'esempio di una statua romana lesionata. Atti 18a Conf. Naz. ASITA 2014, pp. 1229-1236.

Ye, X., Li, J., Huang, H., Du, L., Zhang, X., 2018. 3D Recurrent Neural Networks with Context Fusion for Point Cloud Semantic Segmentation. In: Lecture Notes in Computer Science (Including Subseries Lecture Notes in Artificial Intelligence and Lecture Notes in Bioinformatics), pp. 415-430. https://doi.org/10.1007/978-3-030-01234-2_25 